\title{
Gas-phase Models for Catalysis: Alkane Activation and Olefin Epoxidation by the Triatomic Cation $\mathrm{Ag}_{2} \mathrm{O}^{+}$
}

\author{
Jana Roithováa ${ }^{\mathrm{a}, \mathrm{b}}$ and Detlef Schröder ${ }^{\mathrm{b} *}$ \\ ${ }^{a}$ Department of Organic Chemistry, Charles University, Hlavova 8, 12843 Prague 2, Czech Republic \\ ${ }^{b}$ Institute of Organic Chemistry and Biochemistry, Academy of Sciences of the Czech Republic, Flemingovo náměstí 2, \\ 16610 Praha 6, Czech Republic
}

\section{Supporting Information}

Cartesian coordinates of the computed structures.

$\left[\mathrm{Ag}_{2}\left(\mathrm{NO}_{3}\right)\left(\mathrm{H}_{2} \mathrm{O}\right)_{2}\right]^{+}$

$E(0 \mathrm{~K})=-727.014918$ Hartree

$\mathrm{N}_{\text {imag }}=0$

Standard orientation:

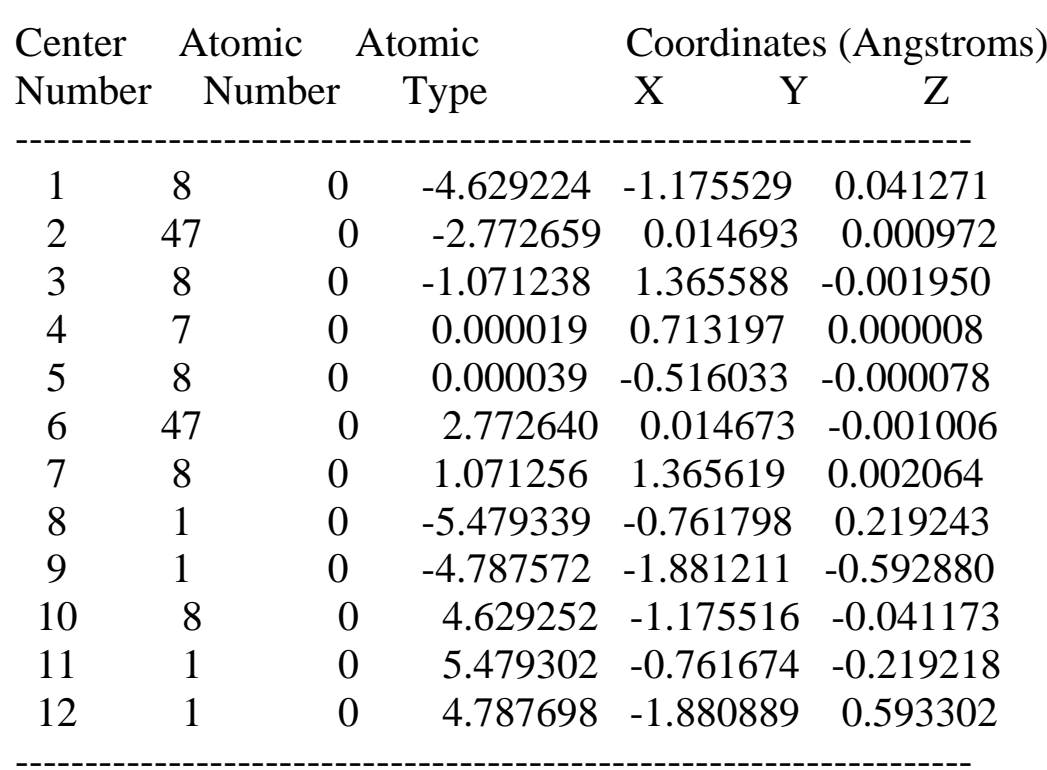


$\left[\mathrm{Ag}_{2}\left(\mathrm{NO}_{3}\right)\left(\mathrm{H}_{2} \mathrm{O}\right)\right]^{+}$

$E(0 \mathrm{~K})=-650.564571$ Hartree

$\mathrm{N}_{\text {imag }}=0$

Standard orientation:

\begin{tabular}{|c|c|c|c|c|c|}
\hline \multirow{2}{*}{$\begin{array}{l}\text { Center } \\
\text { Number }\end{array}$} & \multirow{2}{*}{\multicolumn{2}{|c|}{$\begin{array}{l}\text { Atomic } \\
\text { Number }\end{array}$}} & \multirow{2}{*}{$\begin{array}{r}\text { Atomic } \\
\text { Type }\end{array}$} & \multicolumn{2}{|c|}{ Coordinates (Angstron } \\
\hline & & & & $\mathrm{X} \quad \mathrm{Y}$ & $\mathrm{Z}$ \\
\hline 1 & 8 & 0 & -1.530790 & 1.498984 & 0.004027 \\
\hline 2 & 47 & 0 & -2.905369 & -0.393131 & -0.003069 \\
\hline 3 & 8 & 0 & -0.498916 & -0.397693 & 0.004705 \\
\hline 4 & 47 & 0 & 2.313404 & 0.081793 & 0.001218 \\
\hline 5 & 8 & 0 & 0.621251 & 1.457959 & 0.008465 \\
\hline 6 & 7 & 0 & -0.472009 & 0.857876 & 0.005628 \\
\hline 7 & 8 & 0 & 4.126983 & -1.171216 & -0.026693 \\
\hline 8 & 1 & 0 & 4.830114 & -1.015020 & -0.664755 \\
\hline 9 & 1 & 0 & 4.548054 & -1.461472 & 0.788304 \\
\hline
\end{tabular}

$\left[\mathrm{Ag}_{2}\left(\mathrm{NO}_{3}\right)\right]^{+}$

$E(0 \mathrm{~K})=-574.112394$ Hartree

$\mathrm{N}_{\text {imag }}=0$

Standard orientation:

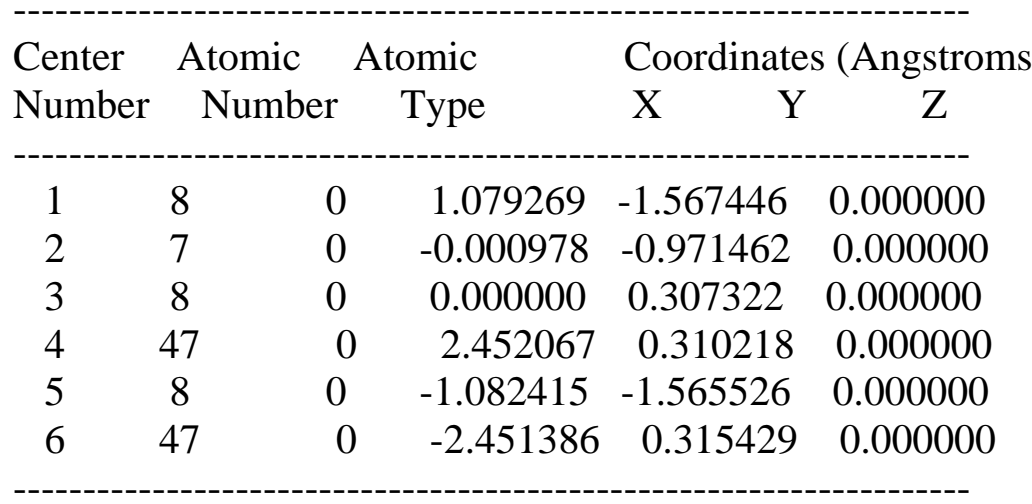

[Ag( $\left.\left(\mathrm{NO}_{3}\right)\right]$

$E(0 \mathrm{~K})=-427.320104$ Hartree

$\mathrm{N}_{\text {imag }}=0$

Standard orientation:

\begin{tabular}{cccccc} 
Center & \multicolumn{2}{c}{ Atomic } & Atomic & \multicolumn{3}{c}{ Coordinates (Angstroms) } \\
Number & Number & Type & X & Y & Z \\
- \hdashline & 8 & 0 & -1.085950 & -0.954728 & 0.000000 \\
2 & 47 & 0 & 0.000000 & 1.048034 & 0.000000 \\
3 & 7 & 0 & -0.000047 & -1.624083 & 0.000000 \\
4 & 8 & 0 & -0.000045 & -2.826778 & 0.000000 \\
5 & 8 & 0 & 1.086035 & -0.954623 & 0.000000 \\
\hline
\end{tabular}


$\left[\mathrm{Ag}_{2} \mathrm{O}\right]^{+}$

$E(0 \mathrm{~K})=-368.941510$ Hartree

$\mathrm{N}_{\text {imag }}=0$

Standard orientation:

Center Atomic Atomic Coordinates (Angstroms)

Number Number Type $\quad$ X $\quad$ Y $\quad$ Z

$\begin{array}{cccccc}1 & 8 & 0 & 0.000000 & 0.003127 & 0.000000 \\ 2 & 47 & 0 & 2.083619 & 0.274390 & 0.000000 \\ 3 & 47 & 0 & -2.083619 & -0.274923 & 0.000000\end{array}$

$\left[\mathrm{Ag}_{2}\right]^{+}$

$E(0 \mathrm{~K})=-293.777032$ Hartree

$\mathrm{N}_{\text {imag }}=0$

Standard orientation:

\begin{tabular}{|c|c|c|c|c|}
\hline Center & Atomic & Atomic & Coordinate & s (Angstron \\
\hline Number & Number & Type & $\mathrm{X}$ & Z \\
\hline 1 & 47 & 0.000000 & 0.000000 & 1.383466 \\
\hline 2 & 47 & 0.000000 & 0.000000 & -1.383466 \\
\hline
\end{tabular}

$\left[\mathrm{Ag}_{2} \mathrm{O} \ldots . . \mathrm{CH}_{4}\right]^{+}$

$E(0 \mathrm{~K})=-409.435650$ Hartree

$\mathrm{N}_{\text {imag }}=0$

Standard orientation:

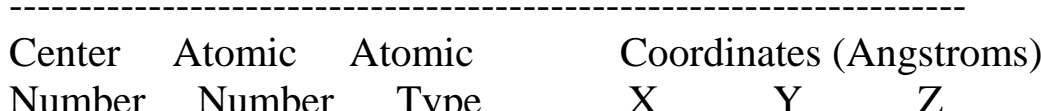

Number Number Type $\quad$ X $\quad$ Y $\quad$ Z

$\begin{array}{cccccc}-1 & 6 & 0 & -4.045064 & 0.288727 & 0.002996 \\ 2 & 47 & 0 & -1.640287 & -0.062630 & -0.001309 \\ 3 & 8 & 0 & 0.395097 & -0.414943 & 0.003428 \\ 4 & 47 & 0 & 2.435454 & 0.071600 & 0.000086 \\ 5 & 1 & 0 & -3.485294 & 0.214831 & -0.952003 \\ 6 & 1 & 0 & -4.776068 & -0.514879 & -0.003892 \\ 7 & 1 & 0 & -3.484873 & 0.198411 & 0.956318 \\ 8 & 1 & 0 & -4.517013 & 1.267214 & 0.011693 \\ -\end{array}$


$\left[\mathrm{Ag}_{2} \mathrm{O} \ldots . . \mathrm{CH}_{4}\right.$ TS H-Transfer] ${ }^{+}$

$E(0 \mathrm{~K})=-409.412607$ Hartree

$\mathrm{N}_{\text {imag }}=1(-943.8307)$

Standard orientation:

\begin{tabular}{|c|c|c|c|c|}
\hline \multirow{2}{*}{$\begin{array}{l}\text { Center } \\
\text { Number }\end{array}$} & \multirow{2}{*}{$\begin{array}{l}\text { Atomic } \\
\text { Number }\end{array}$} & \multirow{2}{*}{$\begin{array}{r}\text { Atomic } \\
\text { Type }\end{array}$} & \multicolumn{2}{|c|}{ Coordinates (Angstrom } \\
\hline & & & $\begin{array}{ll}X & Y\end{array}$ & $\mathrm{Z}$ \\
\hline 1 & 8 & 0.000002 & 0.282358 & -0.466966 \\
\hline 2 & 47 & 1.961275 & -0.315472 & 0.032142 \\
\hline 3 & 47 & -1.961262 & -0.315492 & 0.032141 \\
\hline 4 & 1 & 0.000092 & 1.591173 & -0.212906 \\
\hline 5 & 6 & -0.000065 & 2.769945 & 0.082889 \\
\hline 6 & 1 & 0.901805 & 3.175110 & -0.368450 \\
\hline 7 & 1 & -0.901911 & 3.174915 & -0.368675 \\
\hline 8 & 1 & -0.000208 & 2.835614 & 1.167132 \\
\hline
\end{tabular}

$\left[\mathrm{Ag}_{2} \mathrm{OH} \ldots . . . \mathrm{CH}_{3}\right]^{+}$

$E(0 \mathrm{~K})=-409.463182$ Hartree

$\mathrm{N}_{\text {imag }}=0$

Input orientation:

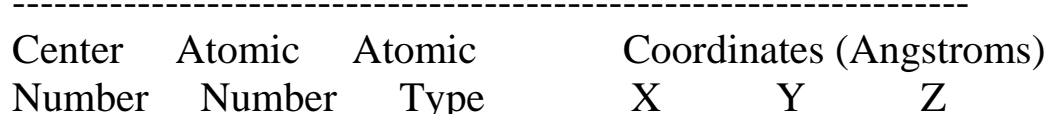

$\begin{array}{cccccc}1 & 6 & 0 & 0.101526 & -0.120126 & 0.113913 \\ 2 & 47 & 0 & 0.112906 & -0.112139 & 2.280473 \\ 3 & 8 & 0 & 0.124568 & -0.104424 & 4.401752 \\ 4 & 47 & 0 & -1.250814 & -0.093007 & 5.998224 \\ 5 & 1 & 0 & 1.065507 & 0.336747 & -0.091307 \\ 6 & 1 & 0 & -0.776749 & 0.486944 & -0.086684 \\ 7 & 1 & 0 & 0.014249 & -1.184398 & -0.085410 \\ 8 & 1 & 0 & 1.019894 & -0.110715 & 4.748214\end{array}$

$\left[\mathrm{Ag}_{2} \mathrm{OH}\right]^{+}$

$E(0 \mathrm{~K})=-369.617538$ Hartree

$\mathrm{N}_{\text {imag }}=0$

Standard orientation:

\begin{tabular}{cccccc} 
Center & \multicolumn{2}{c}{ Atomic } & \multicolumn{2}{c}{ Atomic } & \multicolumn{2}{c}{ Coordinates } & (Angstrom \\
Number & Number & Type & X & Y & Z \\
--------------- \\
1 & 8 & 0 & 0.000134 & 0.640631 & -0.000039 \\
2 & 47 & 0 & 1.998418 & -0.071561 & 0.000002 \\
3 & 47 & 0 & -1.998441 & -0.071552 & 0.000002 \\
4 & 1 & 0 & 0.000027 & 1.601267 & 0.000133
\end{tabular}


$\left[\mathrm{Ag}_{2} \mathrm{O} \ldots . . \mathrm{C}_{2} \mathrm{H}_{6}\right]^{+}$

$E(0 \mathrm{~K})=-448.722413$ Hartree

$\mathrm{N}_{\text {imag }}=0$

Standard orientation:

\begin{tabular}{|c|c|c|c|c|c|}
\hline \multirow{2}{*}{$\begin{array}{l}\text { Center } \\
\text { Number }\end{array}$} & \multirow{2}{*}{$\begin{array}{l}\text { Atomic } \\
\text { Number }\end{array}$} & \multirow{2}{*}{\multicolumn{2}{|c|}{$\begin{array}{r}\text { Atomic } \\
\text { Type }\end{array}$}} & \multicolumn{2}{|c|}{ Coordinates (Angstrom } \\
\hline & & & & $\mathrm{X} \quad \mathrm{Y}$ & $\mathrm{Z}$ \\
\hline 1 & 6 & 0 & 3.679647 & -0.503902 & 0.066810 \\
\hline 2 & 47 & 0 & 1.277888 & -0.063071 & -0.054857 \\
\hline 3 & 8 & 0 & -0.738690 & 0.361992 & -0.188764 \\
\hline 4 & 47 & 0 & -2.778442 & 0.000875 & 0.047170 \\
\hline 5 & 1 & 0 & 3.152392 & -0.402302 & -0.909736 \\
\hline 6 & 6 & 0 & 4.807565 & 0.515113 & 0.140166 \\
\hline 7 & 1 & 0 & 3.038215 & -0.407845 & 0.974948 \\
\hline 8 & 1 & 0 & 4.022705 & -1.537596 & 0.087215 \\
\hline 9 & 1 & 0 & 5.490526 & 0.384265 & -0.698467 \\
\hline 10 & 1 & 0 & 4.433675 & 1.538055 & 0.114458 \\
\hline 11 & 1 & 0 & 5.374777 & 0.385419 & 1.061120 \\
\hline
\end{tabular}

[Ag $\mathrm{Ag}_{2} \mathrm{O} \ldots . \mathrm{C}_{2} \mathrm{H}_{6}$ TS H-Transfer] ${ }^{+}$ $E(0 \mathrm{~K})=-448.702390$ Hartree $\mathrm{N}_{\text {imag }}=1(-507.7288)$

Standard orientation:

\begin{tabular}{|c|c|c|c|c|c|}
\hline \multirow{2}{*}{$\begin{array}{l}\text { Center } \\
\text { Number }\end{array}$} & \multirow{2}{*}{$\begin{array}{l}\text { Atomic } \\
\text { Number }\end{array}$} & \multirow{2}{*}{\multicolumn{2}{|c|}{$\begin{array}{l}\text { Atomic } \\
\text { Type }\end{array}$}} & \multicolumn{2}{|c|}{ Coordinates (Angstroms } \\
\hline & & & & X $\quad Y$ & $\mathrm{Z}$ \\
\hline 1 & 6 & O & -0.581664 & 2.445677 & 0.027208 \\
\hline 2 & 8 & 0 & 0.163731 & 0.000288 & 0.467932 \\
\hline 3 & 47 & 0 & 2.199068 & -0.093492 & -0.042552 \\
\hline 4 & 47 & 0 & -1.718151 & -0.822636 & -0.026276 \\
\hline 5 & 1 & 0 & -0.154261 & 1.366456 & 0.255545 \\
\hline 6 & 1 & 0 & -0.245293 & 3.039539 & 0.876922 \\
\hline 7 & 1 & 0 & -0.091000 & 2.772867 & -0.889108 \\
\hline 8 & 6 & 0 & -2.081221 & 2.356870 & -0.089269 \\
\hline 9 & 1 & 0 & -2.515067 & 3.344828 & -0.270681 \\
\hline 10 & 1 & 0 & -2.390302 & 1.735286 & -0.937932 \\
\hline 11 & 1 & 0 & -2.539723 & 1.981469 & 0.829102 \\
\hline
\end{tabular}


$\left[\mathrm{Ag}_{2} \mathrm{OH} \ldots \mathrm{C}_{2} \mathrm{H}_{5}\right]^{+}$

$E(0 \mathrm{~K})=-448.761713$ Hartree

$\mathrm{N}_{\text {imag }}=0$

Standard orientation:

\begin{tabular}{|c|c|c|c|c|c|}
\hline \multirow{2}{*}{$\begin{array}{l}\text { Center } \\
\text { Number }\end{array}$} & \multirow{2}{*}{$\begin{array}{l}\text { Atomic } \\
\text { Number }\end{array}$} & \multirow{2}{*}{\multicolumn{2}{|c|}{$\begin{array}{l}\text { Atomic } \\
\text { Type }\end{array}$}} & \multicolumn{2}{|c|}{ Coordinates (Angstron } \\
\hline & & & & $\mathrm{X} \quad \mathrm{Y}$ & $\mathrm{Z}$ \\
\hline 1 & 6 & 0 & -3.446249 & 0.016470 & -0.608968 \\
\hline 2 & 47 & 0 & -1.343400 & -0.230513 & -0.032987 \\
\hline 3 & 8 & 0 & 0.683921 & -0.464569 & 0.548512 \\
\hline 4 & 47 & 0 & 2.601697 & 0.169235 & -0.060050 \\
\hline 5 & 1 & 0 & -3.686781 & -1.045521 & -0.651600 \\
\hline 6 & 1 & 0 & -3.261001 & 0.478191 & -1.578476 \\
\hline 7 & 6 & 0 & -4.120094 & 0.832936 & 0.424648 \\
\hline 8 & 1 & 0 & 0.766141 & -1.081080 & 1.279700 \\
\hline 9 & 1 & 0 & -4.125701 & 0.361308 & 1.408173 \\
\hline 10 & 1 & 0 & -3.725325 & 1.847034 & 0.496316 \\
\hline 11 & 1 & 0 & -5.180587 & 0.940238 & 0.136451 \\
\hline
\end{tabular}

$\left[\mathrm{Ag}_{2} \mathrm{O} \ldots . . . \mathrm{CH}_{3} \mathrm{CH}_{2} \mathrm{CH}_{3}\right]^{+}$

$E(0 \mathrm{~K})=-488.010865$ Hartree

$\mathrm{N}_{\text {imag }}=1(-48.8822)$ With the actually used functional, basis set, and the grid, it was not possible to localize a structure without this small imaginary frequency.

Standard orientation:

\begin{tabular}{|c|c|c|c|c|c|}
\hline \multirow{2}{*}{$\begin{array}{l}\text { Center } \\
\text { Number }\end{array}$} & \multirow{2}{*}{\multicolumn{2}{|c|}{$\begin{array}{l}\text { Atomic } \\
\text { Number }\end{array}$}} & \multirow{2}{*}{$\begin{array}{l}\text { Atomic } \\
\text { Type }\end{array}$} & \multicolumn{2}{|c|}{ Coordinates (Angstroms } \\
\hline & & & & X $\quad Y$ & $\mathrm{Z}$ \\
\hline 1 & 6 & 0 & -3.363187 & -0.555923 & -0.074584 \\
\hline 2 & 6 & 0 & -4.433275 & 0.252334 & 0.654629 \\
\hline 3 & 6 & 0 & -5.411872 & 0.907200 & -0.305762 \\
\hline 4 & 47 & 0 & -0.941927 & -0.271332 & -0.057752 \\
\hline 5 & 8 & 0 & 1.108921 & -0.046585 & -0.023063 \\
\hline 6 & 47 & 0 & 3.189388 & 0.169938 & 0.023536 \\
\hline 7 & 1 & 0 & -6.175206 & 1.460428 & 0.241263 \\
\hline 8 & 1 & 0 & -4.908879 & 1.610852 & -0.973218 \\
\hline 9 & 1 & 0 & -5.920656 & 0.164346 & -0.923170 \\
\hline 10 & 1 & 0 & -3.957101 & 1.008895 & 1.282490 \\
\hline 11 & 1 & 0 & -4.964408 & -0.420063 & 1.331986 \\
\hline 12 & 1 & 0 & -2.717139 & -1.069672 & 0.674986 \\
\hline 13 & 1 & 0 & -2.838135 & 0.117669 & -0.794441 \\
\hline 14 & 1 & 0 & -3.770508 & -1.355951 & -0.692921 \\
\hline
\end{tabular}


[Ag $\mathrm{Ag}_{2} \mathrm{O} \ldots \mathrm{CH}_{3} \mathrm{CH}_{2} \mathrm{CH}_{3}$ TS H-Transfer]

$E(0 \mathrm{~K})=-487.991088$ Hartree

$\mathrm{N}_{\text {imag }}=1(-599.7903)$

Standard orientation:

\begin{tabular}{|c|c|c|c|c|c|}
\hline \multirow{2}{*}{$\begin{array}{l}\text { Center } \\
\text { Number }\end{array}$} & \multirow{2}{*}{$\begin{array}{l}\text { Atomic } \\
\text { Number }\end{array}$} & \multirow{2}{*}{\multicolumn{2}{|c|}{$\begin{array}{l}\text { Atomic } \\
\text { Type }\end{array}$}} & \multicolumn{2}{|c|}{ Coordinates (Angstrom } \\
\hline & & & & $\begin{array}{ll}X & Y\end{array}$ & $\mathrm{Z}$ \\
\hline 1 & 6 & 0 & -1.064544 & 1.943633 & 0.043431 \\
\hline 2 & 6 & 0 & -2.457518 & 1.366099 & 0.120495 \\
\hline 3 & 8 & 0 & 0.423420 & -0.118116 & 0.455264 \\
\hline 4 & 47 & 0 & -1.169001 & -1.431407 & -0.034458 \\
\hline 5 & 47 & 0 & 2.394385 & 0.389258 & -0.046232 \\
\hline 6 & 1 & 0 & -0.286768 & 1.070316 & 0.266297 \\
\hline 7 & 1 & 0 & -0.860647 & 2.684884 & 0.817049 \\
\hline 8 & 1 & 0 & -0.806056 & 2.332994 & -0.941747 \\
\hline 9 & 6 & 0 & -3.542174 & 2.410543 & -0.140003 \\
\hline 10 & 1 & 0 & -2.568237 & 0.566545 & -0.634338 \\
\hline 11 & 1 & 0 & -2.619148 & 0.920805 & 1.107471 \\
\hline 12 & 1 & 0 & -4.534522 & 1.962590 & -0.078796 \\
\hline 13 & 1 & 0 & -3.488792 & 3.210141 & 0.600162 \\
\hline 14 & 1 & 0 & -3.430797 & 2.855995 & -1.129331 \\
\hline
\end{tabular}

$\left[\mathrm{Ag}_{2} \mathrm{OH} \ldots . . \mathrm{CH}_{2} \mathrm{CH}_{2} \mathrm{CH}_{3}\right]^{+}$

$E(0 \mathrm{~K})=-488.049868$ Hartree

$\mathrm{N}_{\text {imag }}=0$

Standard orientation:

\begin{tabular}{|c|c|c|c|c|c|}
\hline \multirow{2}{*}{$\begin{array}{l}\text { Center } \\
\text { Number }\end{array}$} & \multirow{2}{*}{$\begin{array}{l}\text { Atomic } \\
\text { Number }\end{array}$} & \multirow{2}{*}{\multicolumn{2}{|c|}{$\begin{array}{l}\text { Atomic } \\
\text { Type }\end{array}$}} & \multicolumn{2}{|c|}{ Coordinates (Angstrom } \\
\hline & & & & X $\quad Y$ & $\mathrm{Z}$ \\
\hline 1 & 6 & 0 & -3.158029 & -0.622186 & -0.614997 \\
\hline 2 & 6 & 0 & -4.027879 & 0.239870 & 0.217261 \\
\hline 3 & 47 & 0 & -1.057985 & -0.442693 & 0.020550 \\
\hline 4 & 8 & 0 & 0.963784 & -0.314102 & 0.664333 \\
\hline 5 & 47 & 0 & 2.846070 & 0.245697 & -0.099735 \\
\hline 6 & 1 & 0 & -3.222085 & -1.699943 & -0.462941 \\
\hline 7 & 1 & 0 & -2.983130 & -0.315034 & -1.647559 \\
\hline 8 & 1 & 0 & 1.065598 & -0.652990 & 1.556639 \\
\hline 9 & 1 & 0 & -3.963618 & -0.047334 & 1.272123 \\
\hline 10 & 6 & 0 & -3.844529 & 1.738843 & 0.030433 \\
\hline 11 & 1 & 0 & -5.061926 & -0.036046 & -0.064261 \\
\hline 12 & 1 & 0 & -4.583984 & 2.290636 & 0.609607 \\
\hline 13 & 1 & 0 & -2.857759 & 2.068683 & 0.363625 \\
\hline 14 & 1 & 0 & -3.960730 & 2.024487 & -1.016384 \\
\hline
\end{tabular}


$\left[\mathrm{Ag}_{2} \mathrm{O} \ldots . . . \mathrm{CH}_{2}\left(\mathrm{CH}_{3}\right)_{2}\right]^{+}$

$E(0 \mathrm{~K})=-488.011138$ Hartree

$\mathrm{N}_{\text {imag }}=1$ (-1.2279) With the actually used functional, basis set, and the grid, it was not possible to localize a structure without this small imaginary frequency.

Input orientation:

\begin{tabular}{|c|c|c|c|c|c|}
\hline \multirow{2}{*}{$\begin{array}{l}\text { Center } \\
\text { Number }\end{array}$} & Atomic & \multicolumn{2}{|c|}{ Atomic } & \multicolumn{2}{|c|}{ Coordinates (Angstron } \\
\hline & Numbe & & Type & X $\quad Y$ & $\mathrm{Z} \quad \mathrm{Z}$ \\
\hline 1 & 6 & 0 & 0.013521 & 0.010925 & -0.005023 \\
\hline 2 & 6 & & -0.010558 & 0.051655 & 1.517500 \\
\hline 3 & 6 & U & 361097 & -0.009493 & 2.176682 \\
\hline 4 & 47 & 0 & 70 & 0.266800 & 2.725630 \\
\hline 5 & 8 & & -3.9 & 0.435 & 3.730766 \\
\hline 6 & 47 & & -5.731897 & 0.612973 & 4.768102 \\
\hline 7 & 1 & & -0.588800 & -0.837305 & 1.871474 \\
\hline 8 & 1 & 0 & -0.4 & 1.029296 & 1.817585 \\
\hline 9 & 1 & 0 & & -0.906470 & -0.348057 \\
\hline 10 & 1 & 0 & -0.9 & 0.050364 & -0.437053 \\
\hline 11 & 1 & & 0.584097 & 0.854112 & -0.395936 \\
\hline 12 & 1 & 0 & 1.873113 & -0.928981 & 1.890737 \\
\hline 13 & 1 & 0 & 1.973365 & 0.831714 & 1.849852 \\
\hline 14 & 1 & 0 & 1.298604 & 0.018965 & 3.264493 \\
\hline
\end{tabular}

$\left[\mathrm{Ag}_{2} \mathrm{O} \ldots \mathrm{CH}_{2}\left(\mathrm{CH}_{3}\right)_{2} \mathrm{TS}\right.$ H-Transfer] ${ }^{+}$

$E(0 \mathrm{~K})=-487.992375$ Hartree

$\mathrm{N}_{\text {imag }}=1(-296.2293)$

Standard orientation:

\begin{tabular}{|c|c|c|c|c|c|}
\hline Center & Atomic & & tomic & Coordinate & es (Angstroms \\
\hline Number & Numbe & & Type & X $\quad Y$ & $\mathrm{Z}$ \\
\hline 1 & 6 & 0 & -0.644052 & 2.194055 & 0.484968 \\
\hline 2 & 6 & 0 & -2.117631 & 1.989345 & 0.203342 \\
\hline 3 & 8 & 0 & 0.184792 & -0.328904 & 0.522513 \\
\hline 4 & 47 & 0 & -1.742989 & -0.967403 & -0.093699 \\
\hline 5 & 47 & 0 & 2.210921 & -0.311008 & -0.014618 \\
\hline 6 & 1 & 0 & -0.155291 & 1.140111 & 0.526354 \\
\hline 7 & 1 & 0 & -0.489472 & 2.566593 & 1.500575 \\
\hline 8 & 6 & 0 & 0.081581 & 3.037625 & -0.537181 \\
\hline 9 & 1 & 0 & -2.659159 & 2.939840 & 0.212817 \\
\hline 10 & 1 & 0 & -2.281885 & 1.564547 & -0.796203 \\
\hline 11 & 1 & 0 & -2.595550 & 1.364206 & 0.966384 \\
\hline 12 & 1 & 0 & -0.342939 & 4.045454 & -0.564503 \\
\hline 13 & 1 & 0 & 1.140622 & 3.146445 & -0.297457 \\
\hline 14 & 1 & 0 & -0.006848 & 2.623208 & -1.543966 \\
\hline
\end{tabular}


$\left[\mathrm{Ag}_{2} \mathrm{OH} . . . . \mathrm{CH}\left(\mathrm{CH}_{3}\right)_{2}\right]^{+}$

$E(0 \mathrm{~K})=-488.058151$ Hartree

$\mathrm{N}_{\text {imag }}=0$

Standard orientation:

\begin{tabular}{|c|c|c|c|c|c|}
\hline \multirow{2}{*}{$\begin{array}{l}\text { Center } \\
\text { Number }\end{array}$} & Atomic & \multicolumn{2}{|c|}{ Atomic } & \multicolumn{2}{|c|}{ Coordinates (Angstroms) } \\
\hline & Numbe & & Type & X $\quad \mathrm{Y}$ & $\mathrm{Z}$ \\
\hline 1 & 6 & 0 & 3.226254 & 0.303461 & -0.397533 \\
\hline 2 & 6 & 0 & 3.420914 & 1.623464 & 0.264370 \\
\hline 3 & 6 & 0 & 3.980607 & -0.876073 & 0.110379 \\
\hline 4 & 1 & 0 & 5.046462 & -0.740197 & -0.134595 \\
\hline 5 & 1 & 0 & 3.927336 & -0.973009 & 1.196386 \\
\hline 6 & 1 & 0 & 3.678735 & -1.813268 & -0.358367 \\
\hline 7 & 1 & 0 & 3.091670 & 0.339698 & -1.482175 \\
\hline 8 & 1 & 0 & 2.735765 & 2.390573 & -0.097953 \\
\hline 9 & 1 & 0 & 3.361081 & 1.560540 & 1.352514 \\
\hline 10 & 1 & 0 & 4.435879 & 1.984038 & 0.031724 \\
\hline 11 & 47 & 0 & 1.096556 & -0.205890 & -0.051344 \\
\hline 12 & 8 & 0 & -0.940762 & -0.693945 & 0.285136 \\
\hline 13 & 1 & 0 & -1.032375 & -1.566663 & 0.674041 \\
\hline 14 & 47 & 0 & -2.830282 & 0.164714 & t -0.019421 \\
\hline
\end{tabular}

$\left[\mathrm{Ag}_{2} \mathrm{O} \ldots . \mathrm{C}_{2} \mathrm{H}_{4}\right]^{+}$

$E(0 \mathrm{~K})=-447.531910$ Hartree

$\mathrm{N}_{\text {imag }}=0$

Input orientation:

\begin{tabular}{|c|c|c|c|c|c|}
\hline Cente & Atomic & & omic & Coordin & \\
\hline Number & Numb & & Type & $\mathrm{X}$ & $\mathrm{Z}$ \\
\hline 1 & 6 & 0 & -0.015451 & 0.005361 & -0.000123 \\
\hline 2 & 6 & 0 & & 0.007572 & 1.356668 \\
\hline 3 & 47 & 0 & 2.171743 & -0.118014 & 0.637273 \\
\hline 4 & 1 & 0 & -0.163923 & -0.913594 & -0.556910 \\
\hline 5 & 1 & 0 & -0.062 & 0.931880 & -0.562089 \\
\hline 6 & 1 & 0 & -0.1 & -0.909818 & 1.921766 \\
\hline 7 & 1 & & -0.014 & 0.935625 & 1.917520 \\
\hline 8 & 8 & 0 & 4.233478 & -0.269598 & 0.629389 \\
\hline 9 & 47 & 0 & 6.274478 & -0.500493 & 0.288019 \\
\hline
\end{tabular}


$\mathrm{TS} \mathbf{1} / \mathbf{2}^{+}$

$E(0 \mathrm{~K})=-447.491884$ Hartree

$\mathrm{N}_{\text {imag }}=1(-248.9988)$

Standard orientation:

\begin{tabular}{|c|c|c|c|c|c|}
\hline Center & Atomic & & tomic & Coordinates & s (Angstrom \\
\hline Number & Numbe & & Type & X $\quad Y$ & $\mathrm{Z}$ \\
\hline 1 & 6 & 0 & -1.832199 & 1.729961 & -0.477929 \\
\hline 2 & 6 & 0 & -0.741248 & 2.101717 & 0.256332 \\
\hline 3 & 1 & 0 & 0.172803 & 2.404366 & -0.235687 \\
\hline 4 & 1 & 0 & -0.827989 & 2.334881 & 1.307808 \\
\hline 5 & 1 & 0 & -2.819524 & 1.728499 & -0.021161 \\
\hline 6 & 1 & 0 & -1.784969 & 1.697434 & -1.561232 \\
\hline 7 & 47 & 0 & -1.637221 & -0.603026 & -0.012838 \\
\hline 8 & 8 & 0 & 0.176560 & 0.190815 & 0.790165 \\
\hline 9 & 47 & 0 & 2.047602 & -0.092331 & -0.082512 \\
\hline
\end{tabular}

[Ag•c-AgOC $\left.{ }_{2} \mathrm{H}_{4}\right]^{+}$

$E(0 \mathrm{~K})=-447.530597$ Hartree

$\mathrm{N}_{\text {imag }}=0$

Input orientation:

\begin{tabular}{|c|c|c|c|c|}
\hline \multirow{2}{*}{$\begin{array}{l}\text { Center } \\
\text { Number }\end{array}$} & \multirow{2}{*}{$\begin{array}{l}\text { Atomic } \\
\text { Number }\end{array}$} & \multirow{2}{*}{$\begin{array}{l}\text { Atomic } \\
\text { Type }\end{array}$} & \multicolumn{2}{|c|}{ Coordinates (Angstrom } \\
\hline & & & $\begin{array}{ll}\mathrm{X} & \mathrm{Y}\end{array}$ & $\mathrm{Z}$ \\
\hline 1 & 6 & 0.276042 & 0.528771 & 0.300309 \\
\hline 2 & 6 & 0.086285 & -0.288631 & 1.534628 \\
\hline 3 & 8 & 1.366629 & -0.297616 & 2.186721 \\
\hline 4 & 47 & 2.467449 & 1.082566 & 0.879852 \\
\hline 5 & 47 & 1.613520 & -1.437206 & 3.951975 \\
\hline 6 & 1 & -0.220388 & -1.307841 & 1.282373 \\
\hline 7 & 1 & -0.669717 & 0.157699 & 2.186068 \\
\hline 8 & 1 & 0.515578 & 0.032205 & -0.635115 \\
\hline 9 & 1 & -0.137922 & 1.530462 & 0.242545 \\
\hline
\end{tabular}


$\mathrm{TS} 2 / 3^{+}$

$E(0 \mathrm{~K})=-447.508934$ Hartree

$\mathrm{N}_{\text {imag }}=1(-294.8569)$

Standard orientation:

\begin{tabular}{|c|c|c|c|c|}
\hline Center & Atomic & Atomic & Coordinate & ingstr \\
\hline Number & r Numbei & r Type & $\mathrm{X}$ & Z \\
\hline 1 & 6 & 0.000218 & 2.209736 & 0.694374 \\
\hline 2 & 8 & 0.000140 & 1.073508 & -0.212659 \\
\hline 3 & 6 & 0.000619 & 3.162639 & -0.406488 \\
\hline 4 & 1 & -0.892299 & 2.235095 & 1.318246 \\
\hline 5 & 1 & 0.892500 & 2.234731 & 1.318597 \\
\hline 6 & 1 & -0.928457 & 3.451687 & -0.878479 \\
\hline 7 & 1 & 0.929992 & 3.451341 & -0.878106 \\
\hline 8 & 47 & -1.465226 & -0.555084 & -0.009650 \\
\hline 9 & 47 & 1.465059 & -0.555451 & -0.009633 \\
\hline
\end{tabular}

$\mathrm{TS} 2 / 4^{+}$

$E(0 \mathrm{~K})=-447.499432$ Hartree

$\mathrm{N}_{\text {imag }}=1(-1110.8530)$

Standard orientation:

\begin{tabular}{|c|c|c|c|c|c|}
\hline \multirow{2}{*}{$\begin{array}{l}\text { Center } \\
\text { Number }\end{array}$} & \multirow{2}{*}{\multicolumn{2}{|c|}{$\begin{array}{l}\text { Atomic } \\
\text { Number }\end{array}$}} & \multirow{2}{*}{$\begin{array}{r}\text { Atomic } \\
\text { Type }\end{array}$} & \multicolumn{2}{|c|}{ Coordinates (Angstron } \\
\hline & & & & X $\quad Y$ & $\mathrm{Z}$ \\
\hline 1 & 6 & 0 & -2.333156 & 2.034411 & -0.289095 \\
\hline 2 & 6 & 0 & -0.933943 & 2.064903 & -0.005126 \\
\hline 3 & 8 & 0 & -0.303812 & 1.063148 & 0.615664 \\
\hline 4 & 47 & 0 & 1.609641 & 0.141402 & -0.049386 \\
\hline 5 & 47 & 0 & -0.982373 & -1.052576 & -0.007547 \\
\hline 6 & 1 & 0 & -0.343795 & 2.704711 & -0.667514 \\
\hline 7 & 1 & 0 & -2.993448 & 1.397231 & 0.288829 \\
\hline 8 & 1 & 0 & -2.767970 & 2.805786 & -0.913794 \\
\hline 9 & 1 & 0 & -1.343307 & 2.816376 & 0.808353 \\
\hline
\end{tabular}


$\left[\mathrm{Ag}_{2}{ }^{\bullet} \mathrm{C}-\mathrm{C}_{2} \mathrm{H}_{4} \mathrm{O}\right]^{+}$

$E(0 \mathrm{~K})=-447.555293$ Hartree

$\mathrm{N}_{\text {imag }}=0$

Standard orientation:

\begin{tabular}{|c|c|c|c|c|c|}
\hline \multirow{2}{*}{$\begin{array}{l}\text { Center } \\
\text { Number }\end{array}$} & \multirow{2}{*}{\multicolumn{2}{|c|}{$\begin{array}{l}\text { Atomic } \\
\text { Number }\end{array}$}} & \multirow{2}{*}{$\begin{array}{r}\text { Atomic } \\
\text { Type }\end{array}$} & \multicolumn{2}{|c|}{ Coordinates (Angstroms } \\
\hline & & & & $\begin{array}{ll}X & Y\end{array}$ & $\mathrm{Z}$ \\
\hline 1 & 6 & 0 & -3.831007 & -0.725730 & 0.236041 \\
\hline 2 & 8 & 0 & -2.702867 & 0.000210 & -0.300706 \\
\hline 3 & 47 & 0 & 2.257253 & 0.001306 & 0.056697 \\
\hline 4 & 47 & 0 & -0.477911 & -0.002503 & -0.092360 \\
\hline 5 & 6 & 0 & -3.828829 & 0.732304 & 0.232222 \\
\hline 6 & 1 & 0 & -4.392423 & -1.261838 & -0.518697 \\
\hline 7 & 1 & 0 & -3.634892 & -1.253146 & 1.161436 \\
\hline 8 & 1 & 0 & -4.388634 & 1.266123 & -0.525334 \\
\hline 9 & 1 & 0 & -3.631167 & 1.263987 & 1.154843 \\
\hline
\end{tabular}

$\left[\mathrm{Ag}_{2} \bullet \mathrm{CH}_{3} \mathrm{CHO}\right]^{+}$

$E(0 \mathrm{~K})=-447.602807$ Hartree

$\mathrm{N}_{\text {imag }}=0$

Standard orientation:

\begin{tabular}{|c|c|c|c|c|}
\hline \multirow{2}{*}{$\begin{array}{l}\text { Center } \\
\text { Number }\end{array}$} & \multirow{2}{*}{$\begin{array}{l}\text { Atomic } \\
\text { Number }\end{array}$} & \multirow{2}{*}{$\begin{array}{r}\text { Atomic } \\
\text { Туре }\end{array}$} & \multicolumn{2}{|c|}{ Coordinates (Angstrom } \\
\hline & & & $\begin{array}{ll}X & Y\end{array}$ & $\mathrm{Z}$ \\
\hline 1 & 6 & 4.964557 & -0.038512 & 0.000060 \\
\hline 2 & 6 & 3.577416 & 0.458552 & -0.000091 \\
\hline 3 & 8 & 2.605143 & -0.278753 & -0.000057 \\
\hline 4 & 47 & 0.394492 & -0.077988 & -0.000026 \\
\hline 5 & 47 & -2.341367 & 0.047072 & 0.000021 \\
\hline 6 & 1 & 5.004434 & -1.124637 & -0.001529 \\
\hline 7 & 1 & 5.484139 & 0.366778 & 0.874441 \\
\hline 8 & 1 & 5.485832 & 0.369605 & -0.871943 \\
\hline 9 & 1 & 3.435761 & 1.551085 & -0.000113 \\
\hline
\end{tabular}


$\left[\mathrm{Ag} \cdot \mathrm{C}-\mathrm{C}_{2} \mathrm{H}_{4} \mathrm{O}\right]^{+}$

$E(0 \mathrm{~K})=-300.508369$ Hartree

$\mathrm{N}_{\text {imag }}=0$

Standard orientation:

\begin{tabular}{|c|c|c|c|c|c|}
\hline Center & Atomic & & Atomic & Coordinate & es (Angstro \\
\hline Number & Numbe & & Type & $\mathrm{X}$ & Z \\
\hline 1 & 8 & 0 & 1.160333 & -0.000146 & -0.348397 \\
\hline 2 & 47 & 0 & -1.005310 & -0.000005 & 0.014572 \\
\hline 3 & 6 & 0 & 2.326870 & 0.729050 & 0.113756 \\
\hline 4 & 6 & 0 & 2.327122 & -0.728893 & 0.113816 \\
\hline 5 & 1 & & 2.191489 & -1.257901 & 1.048921 \\
\hline 6 & 1 & 0 & 2.830412 & -1.265219 & -0.680390 \\
\hline 7 & 1 & 0 & 2.829981 & 1.265492 & -0.680485 \\
\hline 8 & 1 & 0 & 2.191058 & 1.258070 & 1.048828 \\
\hline
\end{tabular}

$\left[\mathrm{Ag} \cdot \mathrm{CH}_{3} \mathrm{CHO}\right]^{+}$

$E(0 \mathrm{~K})=-300.556812$ Hartree

$\mathrm{N}_{\text {imag }}=0$

Standard orientation:

\begin{tabular}{|c|c|c|c|c|c|}
\hline \multirow{2}{*}{$\begin{array}{l}\text { Center } \\
\text { Number }\end{array}$} & Atomic & \multirow{2}{*}{\multicolumn{2}{|c|}{$\begin{array}{l}\text { Atomic } \\
\text { Type }\end{array}$}} & \multicolumn{2}{|c|}{ Coordinates (Angstron } \\
\hline & Numbe & & & X $\quad Y$ & $\mathrm{Z}$ \\
\hline 1 & 8 & 0 & 1.020661 & -0.293460 & -0.000066 \\
\hline 2 & 47 & & -1.144113 & 0.001386 & 0.000007 \\
\hline 3 & 6 & & 2.024978 & 0.407605 & -0.000057 \\
\hline 4 & 6 & 0 & 3.384587 & -0.150564 & -0.000005 \\
\hline 5 & 1 & 0 & 48 & -1.237207 & -0.001119 \\
\hline 6 & 1 & & 28 & 0.235960 & 0.873475 \\
\hline 7 & 1 & & 3.922241 & 0.237997 & -0.871787 \\
\hline 8 & 1 & 0 & 1.927800 & 1.503516 & -0.000018 \\
\hline
\end{tabular}


Complete reference 14

Frisch, M. J.; Trucks, G. W.; Schlegel, H. B.; Scuseria, G. E.; Robb, M. A.; Cheeseman, J. R.;

Montgomery, J. A., Jr.; Vreven, T.; Kudin, K. N.; Burant, J. C.; Millam, J. M.; Iyengar, S. S.; Tomasi, J.; Barone, V.; Mennucci, B.; Cossi, M.; Scalmani, G.; Rega, N.; Petersson, G. A.; Nakatsuji, H.; Hada, M.; Ehara, M.; Toyota, K.; Fukuda, R.; Hasegawa, J.; Ishida, M.; Nakajima, T.; Honda, Y.; Kitao, O.; Nakai, H.; Klene, M.; Li, X.; Knox, J. E.; Hratchian, H. P.; Cross, J. B.; Bakken, V.; Adamo, C.; Jaramillo, J.; Gomperts, R.; Stratmann, R. E.; Yazyev, O.; Austin, A. J.; Cammi, R.; Pomelli, C.; Ochterski, J. W.; Ayala, P. Y.; Morokuma, K.; Voth, G. A.; Salvador, P.; Dannenberg, J. J.; Zakrzewski, V. G.; Dapprich, S.; Daniels, A. D.; Strain, M. C.; Farkas, O.; Malick, D. K.; Rabuck, A. D.; Raghavachari, K.; Foresman, J. B.; Ortiz, J. V.; Cui, Q.; Baboul, A. G.; Clifford, S.; Cioslowski, J.; Stefanov, B. B.; Liu, G.; Liashenko, A.; Piskorz, P.; Komaromi, I.; Martin, R. L.; Fox, D. J.; Keith, T.; Al-Laham, M. A.; Peng, C. Y.; Nanayakkara, A.; Challacombe, M.; Gill, P. M. W.; Johnson, B.; Chen, W.; Wong, M. W.; Gonzalez, C.; Pople, J. A. Gaussian 03, revision C.02; Gaussian, Inc.: Wallingford, CT, 2004. 